\title{
Philonsorbonne
}

\section{En avons-nous fini avec l'histoire des mentalités ?}

Florence HULAK

\section{OpenEdition}

\section{Journals}

Édition électronique

URL : https://journals.openedition.org/philonsorbonne/173

DOI : $10.4000 /$ philonsorbonne.173

ISSN : 2270-7336

\section{Éditeur}

Publications de la Sorbonne

\section{Édition imprimée}

Date de publication : 15 avril 2008

Pagination : 89-109

ISBN : 978-2-85944-602-4

ISSN : 1255-183X

\section{Référence électronique}

Florence HULAK, «En avons-nous fini avec l'histoire des mentalités ? », Philonsorbonne [En ligne], 2 | 2008, mis en ligne le 28 janvier 2013, consulté le 08 juin 2021. URL : http://journals.openedition.org/ philonsorbonne/173 ; DOl : https://doi.org/10.4000/philonsorbonne.173

(C) Tous droits réservés 


\title{
En avons-nous fini avec l'histoire des mentalités ?
}

\author{
Florence Hulak
}

L'histoire des mentalités désigne, de façon très générale, l'histoire des formes de pensées, de croyances et de sentiments spécifiques à chaque époque, en tant qu'elles constituent ensemble une appréhension du monde dotée d'une certaine cohérence. Elle représente une part importante de l'œuvre de Marc Bloch et Lucien Febvre, dans les années 1920-30. Après avoir été éclipsée par l'histoire économique et sociale, elle réapparaît massivement dans les années 1960-70, avant de s'effacer peu à peu. L'ouvrage de G. E. R. Lloyd, Pour en finir avec les mentalités ${ }^{\text {, fournit de }}$ la notion de mentalité une critique considérée comme radicale et qui sert fréquemment à invalider l'histoire des mentalités ${ }^{2}$. Or, parce que les historiens avaient déjà, dans une large mesure, cessé d'utiliser cette notion et que son indétermination théorique la rendait difficile à soutenir, peu de voix se sont élevées pour défendre l'histoire des mentalités contre des accusations qui ne l'atteignaient pas véritablement.

Nous montrerons, dans un premier temps, que la confusion des discours portant sur l'histoire des mentalités s'explique notamment par l'occultation de la différence entre deux héritages théoriques incompatibles, celui de Lucien Febvre et celui de Marc Bloch. Puis nous mettrons en évidence l'unité théorique minimale qui subsiste entre ces différentes approches : celle d'une stratégie d'intégration de l'histoire dans le champ contemporain des sciences humaines, voire d'une tentative d'assimilation de ces dernières. Une fois mise en évidence l'unité fondamentale et les oppositions théoriques qui ont constitué l'histoire des mentalités, nous serons alors en mesure d'examiner la validité de la critique de Lloyd à son égard, qui concerne tant sa possible fonction explicative que sa pertinence descriptive.

1. G. E. R. Lloyd, Pour en finir avec les mentalités, trad. Franz Regnot, Paris, La Découverte/ Poche, 1996. Demystifying Mentalities, Cambridge University Press, 1990.

2. Ainsi, c'est la mobilisation de cette référence qui permet à Paul Ricœur d'affirmer la nécessité de passer de l'histoire des mentalités à celle des représentations, in La mémoire, l'histoire, l'oubli, Paris, Le Seuil, 2000, p. 251-253; ou à Jean-Jacques Wunenburger de passer de l'histoire des mentalités à celle de l'imaginaire, in L'imaginaire, Paris, PUF, 2003, p. 6 . 


\section{1/ La notion d'« histoire des mentalités » peut-elle être clarifiée ?}

Si les études portant sur la notion d'histoire des mentalités s'accordent sur un point, c'est bien sur son indétermination. En effet, elle se présente tantôt comme une forme de psychologie collective, tantôt comme une étude de type sociologique ; tantôt comme applicable à tous les paliers de l'analyse historique, tantôt au seul niveau des structures et du temps long. Quelle peut être la cohérence d'un domaine historiographique incluant entre autres l'étude de sentiments (l'amour ${ }^{3}$, la peur ${ }^{4}$ ), de croyances (le diable ${ }^{5}$, le purgatoire $^{6}$ ), d'institutions sociales (le lien féodal ${ }^{7}$, l'impôt $^{8}$, etc.), et de pratiques culturelles (la lecture ${ }^{9}$, etc.) ? Faut-il pour autant renoncer à définir l'histoire des mentalités, en en concluant qu'elle "pointe moins un objet rigoureux qu'elle ne manifeste une sensibilité d'historien ${ }^{10}$ ? Avant de s'y résoudre, il faut préciser la nature de cette indétermination. Est-elle le signe d'une notion plurivoque, c'est-à-dire d'une notion générique, regroupant plusieurs espèces différentes et complémentaires d'histoires des mentalités ? Cette notion est-elle plutôt équivoque, c'est-à-dire l'enjeu d'un conflit entre des approches historiographiques rivales, essayant chacune d'imposer une définition exclusive de l'histoire des mentalités ? S'agit-il au contraire d'une notion irréductiblement confuse, renvoyant à une multiplicité de significations contradictoires, dont aucune ne peut fonder à elle seule une approche cohérente?

\section{a) L'histoire des mentalités de Lucien Febvre et de Marc Bloch : deux pratiques pour un seul terme}

Dès son introduction dans le champ de l'historiographie, la notion d'histoire des mentalités est d'emblée marquée d'une dualité de sens. Bien que l'usage de ce terme soit commun à Marc Bloch et à Lucien Febvre, les deux historiens l'interprètent de façon divergente. L'exploration des mentalités représente pour Febvre un exercice de psychologie historique. C'est à un psychologue, Charles Blondel, qu'il emprunte le terme de

3. J.-L. Flandrin, Les amours paysannes: amour et sexualité dans les campagnes de l'ancienne France, XVI ${ }^{e}$-XIX ${ }^{e}$ siècle, Paris, Julliard, 1975.

4. G. Delumeau, La Peur en Occident : XIV-XVIII ${ }^{e}$, une Cité assiégée, Paris, Fayard, 1978.

5. R. Mandrou, Magistrats et sorciers en France au XVII siècle, Paris, Plon, 1968.

6. J. Le Goff, La naissance du purgatoire, Paris, Gallimard, 1981.

7. G. Duby, «La féodalité ? Une mentalité médiévale », Annales E.S.C, n 13, Paris, 1958, p. 765-777.

8. G. Duby, Guerriers et paysans, «Les attitudes mentales », Paris, Gallimard, 1978.

9. F. Furet et J. Ozouf, Lire et Écrire. L'alphabétisation des français de Calvin à Jules Ferry, Paris, Minuit, 1977.

10. J. Revel, «Mentalités », in A. Burguières (dir.), Dictionnaire des sciences historiques, Paris, PUF, p. 456. 
«mentalité », tiré de l'œuvre de Lévy-Bruhl ${ }^{11}$. Le terme de mentalité désigne alors le système de représentations d'une époque, défini comme un ensemble de catégories de la sensibilité, de l'expression et de la conceptualisation, dont est supposée la cohérence interne. Cet univers mental collectif n'est toutefois accessible que par voie régressive, à partir des réalisations individuelles. D'où le privilège méthodologique de la biographie historique $^{12}$, dans laquelle Febvre entend remonter d'une œuvre à ses conditions de possibilité. Ainsi l'œuvre de Rabelais peut-elle, en raison de sa richesse exceptionnelle, devenir le médium d'une étude de $l^{\prime}$ '« outillage mental » des individus du $\mathrm{XVI}^{\mathrm{e}}$ siècle, c'est-à-dire des éléments linguistiques, intellectuels et affectifs qui circonscrivent le champ de formation de leurs pensées et sentiments individuels. Febvre s'intéresse aux conditions sociales de l'œuvre de Rabelais, en tant qu'elles la déterminent de façon décisive. Mais l'œuvre d'un «grand auteur» comme Rabelais ne constitue pas seulement une source brute de matériaux pour l'étude de l'outillage mental de son époque : elle en manifeste surtout la synthèse effective. Elle montre comment ces différents éléments participent à la formation d'une vision du monde cohérente. Or, la psychologie individuelle de Rabelais étant «celle d'un libre esprit, [...] d'un homme de robuste intelligence, de vigoureux bon sens et dégagé de maints préjugés qui avaient cours autour de lui $»^{13}$, on peut supposer qu'il est allé au bout des possibilités de remise en cause des croyances religieuses offertes par le cadre mental de son époque. Les limites de son incroyance nous renseignent donc sur les limites de l'incroyance possible au $\mathrm{XVI}^{\mathrm{e}}$ siècle. La cohérence de sa vision du monde permet donc de délimiter (ou du moins participe fortement à délimiter) l'univers des possibles dans lequel se déploient les représentations mentales des hommes du XVI ${ }^{\mathrm{e}}$ siècle.

L'histoire des mentalités adopte ainsi la perspective de l'analyse d'une interaction permanente entre la pensée individuelle et son milieu social, voire civilisationnel. Même si Febvre accorde au collectif la primauté sur l'individu, l'unité des différents aspects d'une mentalité - entité tant intellectuelle qu'affective - ne peut se réaliser que dans l'individu. «Que l'historien s'installe au carrefour où toutes les influences viennent se recouper et se fondre : dans la conscience des hommes vivant en société ${ }^{14}$. Si les mentalités sont collectives, l'histoire des mentalités n'en doit pas moins prendre les individus pour centre de son étude. Et tout

11. L. Lévy-Bruhl, Les fonctions mentales dans les sociétés inférieures, Paris, PUF, 1910. La Mentalité Primitive, Paris, Alcan, 1927.

12. L. Febvre, Un destin : M. Luther, Paris, PUF, 1968 [1928]. Rabelais ou le problème de l'incroyance au XVI siècle, Paris, Albin Michel, 1968 [1942]. Amour sacré, amour profane, autour de l'Heptaméron, Paris, Gallimard, 1971, [1944] (sur Marguerite de Navarre).

13. L. Febvre, Rabelais ou le problème de l'incroyance au XVI siècle, op. cit., p. 424.

14. L. Febvre, Projet d'enseignement pour le Collège de France, Paris, 1928, cité par A. Burguières, in «La notion de "mentalité" chez Marc Bloch et Lucien Febvre: deux conceptions, deux filiations », Revue de synthèse, n 111-112, Paris, juill.-déc. 1983, p. 340. 
particulièrement les membres des élites créatrices, dans la mesure où, plus les individus sont démunis, plus leur accès à l'outillage mental de leur époque sera partiel, et moins leur conscience sera en mesure de refléter la richesse et la complexité de la mentalité à laquelle ils appartiennent. Bien que l'ensemble des individus d'une société aient théoriquement accès au même outillage mental, la plupart ne possèdent de fait qu'un nombre réduit d'instruments linguistiques et conceptuels. Par conséquent, leur univers mental personnel sera nécessairement plus pauvre que celui des rares individus qui possèdent la totalité de l'outillage mental mis à disposition par leur époque. La mentalité collective est alors principalement accessible par le biais de l'étude du langage, de l'iconographie artistique et de la littérature ${ }^{15}$. Même si Febvre s'intéresse au conditionnement social des œuvres intellectuelles et culturelles, ce processus reste pensé comme une logique globale : l'inscription des psychologies individuelles dans le cadre d'une psychologie collective, à l'intérieur de laquelle on ne trouve qu'une hiérarchisation sociale de l'accès à la mentalité commune et non une différenciation sociologique des types de pensées et de représentations.

De son côté, Marc Bloch prend d'emblée ses distances avec l'approche individualiste de Febvre, pour étudier les systèmes de croyances et les représentations collectives à partir d'une analyse des rites et des pratiques symboliques qui ne les sépare jamais de leur substrat social. Son ouvrage intitulé Les Rois thaumaturges ${ }^{16}$ prend pour objet la croyance en la puissance guérisseuse des rois, qu'il tente de comprendre comme une réalité sociale. L'analyse statistique de la fréquence des «touchers » royaux, du nombre de participants et de leurs origines géographiques permet de rattacher la croyance à son contexte socio-politique, alors que «les textes littéraires, les documents officiels ne nous offrent de ce sentiment qu'une image déformée, en tout cas suspecte ${ }^{17}$. Et même si cet ouvrage reste traversé d'une dualité entre les «éléments psychologiques profonds ${ }^{18}$ (une croyance en la sacralité des rois, portée par des mythes et légendes ancestraux, qui reste stable pendant des siècles), et les phénomènes socio-politiques «plus fortuits ${ }^{19}$, la suite de l'œuvre de Bloch ira toujours dans le sens d'une spécification sociologique de l'origine des représentations. Ainsi rend-il raison, dans $L a$ Société féodale ${ }^{20}$, du mépris de la vie au Moyen Age par la domination de l'idéologie nobiliaire, dans la mesure où cette dernière compte l'utilisation de la violence parmi ses traits fondamentaux: les chevaliers sont des «hommes pour qui l'audace et le mépris de la vie sont,

15. L. Febvre, «Comment reconstituer la vie mentale d'autrefois? » [1941] repris in Combats pour l'histoire, Paris, Armand Colin, 1992.

16. M. Bloch, Les Rois thaumaturges, Paris, Gallimard, 1983, [1924].

17. Ibid., p. 102.

18. Ibid., p. 114.

19. Ibid., p. 76.

20. M. Bloch, La Société féodale, Paris, Albin Michel, 1994 [1939-1940], tome II, livre 1, chap. 2 : «la vie noble». 
en quelque sorte, des valeurs professionnelles $»^{21}$. Or, dans le compte rendu que Febvre consacre à cette euvre $^{22}$, il prend acte d'une phrase de Bloch mentionnant, parmi les raisons de la violence, le peu d'importance accordée à la vie terrestre perçue comme «état transitoire avant l'éternité », pour suggérer que «la remarque ne vaut pas d'être ainsi jetée négligemment, mais atteste du moins un souci toujours éveillé de mettre en pleine lumière [...] l'action du sentiment religieux sur la conduite des hommes $»^{23}$. Pourtant, si Bloch a jeté «négligemment » cette remarque, c'est qu'il n'accorde pas aux sentiments religieux de réel pouvoir causal: ces derniers se contentent d'accompagner, au niveau des représentations conscientes, une structuration idéologique globale de la société, à laquelle la conscience n'a pas directement accès. L'idéologie religieuse ne doit toutefois pas être pensée comme la simple «cause » des attitudes religieuses. Entre les idéologies et les mentalités, Bloch ne cherche pas de relation de causalité, mais des corrélations synchroniques.

C'est pourquoi l'approche de Bloch ne se caractérise pas exclusivement par l'étude des idéologies et de leur transmission sociale. Il accorde une attention toute particulière à l'analyse de structures synchroniques (sociales, politiques, juridiques, et même aux structures de la parentée ${ }^{24}$ ). En raison du privilège accordé à l'étude des logiques sociales inconscientes sur la diffusion idéologique, l'histoire des mentalités pratiquée par Marc Bloch n'est pas tant sociologique qu'anthropologique. On peut en effet définir «l'anthropologie historique » par l'étude des «phénomènes digérés et intériorisés par la société $»^{25}$, c'est-à-dire de phénomènes indépendants des volontés politiques ou intentions stratégiques des différents groupes sociaux. Les mentalités désignent alors les représentations devenues des habitudes affectives et/ou intellectuelles, mais aussi corporelles. Pour reprendre l'exemple des rois thaumaturges, on peut certes analyser la diffusion idéologique de la croyance dans le pouvoir guérisseur des rois, qui est passée notamment par les traités médicaux. Mais cette idéologie politique n'a eu de prise que dans la mesure où elle se fondait sur un système symbolique, un réseau de «représentations intellectuelles et sentimentales ${ }^{26}$ qui assurait la croyance en la sacralité des rois de France ${ }^{27}$. Les premiers rois à se présenter comme guérisseurs adhéraient eux-mêmes à cette croyance et ne sont parvenus à imposer cette représentation que dans

21. Ibid., p. 410.

22. L. Febvre, «La Société féodale : une synthèse critique », Annales d'histoire économique et sociale, t. 3, Paris, juill.-déc. 1941.

23. Ibid., p. 29.

24. M. Bloch, La Société féodale, op. cit., «La structure du lignage », p. 200 sq.

25. A. Burguières, «L'anthropologie historique », in J. Le Goff (dir.), La Nouvelle histoire, Paris, Complexe, 1988 [1978], p. 145.

26. M. Bloch, Les Rois thaumaturges, op. cit., p. 258.

27. Ibid., p. 52 sq. 
la mesure où il ne s'agissait que de la «cristallisation $»^{28}$ d'une croyance héritée. La diffusion idéologique n'est possible que sur fond d'une structuration symbolique qui lui préexiste. Et lorsque Bloch entreprend d'attribuer des causes aux représentations collectives, ce sont alors les conditions de vie (matérielles, sociales, économiques, démographiques...) qui sont mentionnées. Par exemple, Bloch suppose que les catastrophes variées (épidémies, famines, guerres) de la période féodale «donnaient à l'existence humaine comme un goût de perpétuelle précarité. Là fut probablement une des raisons majeures de l'instabilité des sentiments, si caractéristique de la mentalité de l'ère féodale ${ }^{29}$. Mais cette détermination de la mentalité par les conditions de vie reste incertaine («probablement») et ce mode d'explication n'est jamais utilisé de façon systématique. Les mentalités ne sont donc ni réductibles à l'utilisation idéologique des représentations, ni à leur détermination par les conditions de vie: les mentalités sont des systèmes de représentations non conscientes qui ont leur propre logique, une logique qui constitue la vie sociale.

On peut donc conclure que la notion de mentalité est «psychologique » chez Febvre, alors qu'elle est «sociologique » voire «anthropologique » chez Bloch. Il ne faut toutefois pas se méprendre sur cette opposition. L'étude des mentalités n'exclut pas l'analyse sociologique chez Febvre. Mais on peut produire une description cohérente de la mentalité d'une époque, indépendamment de toute analyse sociologique: cette dernière permet seulement de déterminer la possibilité d'accès des différents individus aux outils mentaux communs. Les mentalités désignent donc chez Febvre une strate ontologique au moins partiellement autonome, le niveau collectif de la psychologie. La tension centrale de l'histoire des mentalités s'institue entre l'autonomie de la conscience individuelle et l'influence reçue de la mentalité collective. Inversement, l'approche « sociologique » de Bloch n'exclut pas directement la psychologie. Mais s'il prétend lui aussi pratiquer la psychologie historique, cette notion ne reconduit pas tant à la notion vague d'univers mental collectif qu'à la dimension impersonnelle des représentations individuelles. Il n'y a donc pas de tension entre l'individu et la mentalité collective: la mentalité est cette réalité non consciente qui structure les représentations les plus personnelles et conscientes. Elle est une réalité trans-individuelle : la conscience passe au rang d'épiphénomène, sur fond de processus essentiellement sociaux. Ainsi, d'un côté, les «mentalités» désignent pour Lucien Febvre le niveau relativement autonome de la psychologie collective. Il y a une existence collective de la pensée et de la sensibilité qui influence sans les déterminer la pensée et la sensibilité des individus. De l'autre, les «mentalités» désignent pour Bloch les logiques non conscientes de la vie matérielle et des représentations collectives, dont on doit rendre raison de façon essentiellement sociologique ou anthropologique.

28. Ibid., p. 81.

29. M. Bloch, La Société féodale, op. cit., p. 116. 
Or, l'héritage de ces deux conceptualisations concomitantes de la notion de mentalité est asymétrique : on doit principalement à l'œuvre de Febvre la paternité théorique de la notion d'histoire des mentalités, alors que ce sont les orientations pratiques des travaux de Bloch qui ont été très majoritairement poursuivies. En effet, Lucien Febvre n'a pas seulement introduit la notion de mentalité en histoire, il s'est aussi efforcé d'en assurer la conceptualisation et la diffusion. Ses deux articles manifestes ${ }^{30}$ sont les premiers textes théoriques consacrés à la notion d'histoire des mentalités. Mais la pratique febvrienne de l'histoire des mentalités, centrée sur la biographie des grands hommes, a eu peu de successeurs. À l'inverse, l'histoire des mentalités qui renaît massivement au début des années 1960, après la longue éclipse qui suit les travaux des deux fondateurs des Annales, s'inscrit sans conteste dans la lignée des travaux de Bloch. Il s'agit d'une forme d'anthropologie historique qui prend pour objet des phénomènes collectifs et ravale la conscience individuelle au rang de phénomène dérivé. Cet héritage asymétrique semble avoir fortement contribué à rendre indistinctes ces deux conceptions de l'histoire des mentalités, favorisant ainsi l'émergence d'une notion unique et équivoque. Dans la mesure où Bloch et Febvre proposaient des analyses différentes de phénomènes analogues, on aurait pu envisager que la notion d'histoire des mentalités soit construite de manière à englober ces deux méthodes différentes de réalisation d'un même objectif. Mais cette dualité n'est pas apparue distinctement, dans la mesure où ces deux perspectives ne se sont pas développées sur le même plan. Si on étudie les textes théoriques élaborés par les historiens, on aura tendance à identifier l'histoire des mentalités à sa définition febvrienne. Mais si on privilégie leur pratique effective, c'est la conception blochienne de l'histoire des mentalités qui apparaît comme seule pertinente. Dès lors, deux définitions de l'histoire des mentalités ont pu se faire concurrence.

\section{b) «L'histoire des mentalités » des années 1960 : une problématique réunification}

Les deux textes théoriques fondateurs de la nouvelle histoire des mentalités des années 1960 - ceux de Georges Duby ${ }^{31}$ et de Robert Mandrou $^{32}$ - sont ainsi conduits, dans leur recherche d'appuis théoriques, à privilégier les orientations de Lucien Febvre. Cette perspective apparaît de façon particulièrement nette dans le texte de Duby : Lucien Febvre y est présenté comme le seul inventeur de l'histoire des mentalités et ses deux articles comme l'unique soubassement théorique de cette histoire. Pour

30. L. Febvre, «Histoire et psychologie » [1938], et «Comment reconstituer la vie mentale d'autrefois ? " [1941] repris in Combats pour l'histoire, Paris, Armand Colin, 1992.

31. G. Duby, «Histoire des mentalités », in L'histoire et ses méthodes, Paris, Gallimard, 1961, p. 937-966.

32. R. Mandrou, «L'histoire des mentalités», in article «Histoire», 5, Encyclopaedia Universalis, vol. VIII, 1968, p. 436-438. 
définir l'histoire des mentalités, on pourrait s'arrêter à ces deux articles, écrit-il, «si les récents progrès des sciences de l'homme n'obligeaient à prolonger ici et là les perspectives qu'ils ouvrent ${ }^{33}$. L'histoire des mentalités ne serait qu'un approfondissement du domaine de recherche ouvert et conceptualisé par Febvre. Elle aurait pour objet le rapport dialectique entre les représentations de l'individu et celles qui lui sont imposées par son milieu.

À l'inverse, lorsqu'on étudie, non plus les textes théoriques, mais la pratique des historiens français dans les années soixante à soixante-dix, c'est la définition anthropologique de l'histoire des mentalités qui s'impose avec évidence. Si on s'intéresse aux travaux des historiens de cette période, comme le fait Roger Chartier $^{34}$, et que l'on étudie une histoire des mentalités «plus exercée que théorisée ${ }^{35}$, la définition de l'histoire des mentalités semble se simplifier: les historiens des mentalités sont « réunis dans une définition de l'histoire des mentalités moins floue qu'on ne l'a dit $»^{36}$. Mais cette simplification va dans un tout autre sens que celui des textes théoriques de la même période, à savoir dans le sens d'une approche anthropologique de l'histoire, armée d'une méthode statistique qui disqualifie l'étude qualitative des œuvres.

Or, cette coexistence de deux définitions hétérogènes de l'histoire des mentalités conduit à la confusion, dans la mesure où chacune des deux approches s'efforce de résorber cette dualité par l'annexion de l'approche concurrente. Ainsi, dans la mesure où Roger Chartier définit l'histoire des mentalités en général d'après la pratique des historiens des années 1960-70, il est contraint de faire dériver cette pratique de l'héritage théorique pourtant hétérogène de Lucien Febvre. Chartier est donc amené à soutenir que les travaux de Febvre préparaient ceux de l'anthropologie historique, que «la voie était ouverte (une fois abandonné le goût particulier de Febvre pour la biographie) $»^{37}$, à l'histoire des mentalités telle qu'elle est pratiquée dans les années soixante. Or, on a vu précédemment que la pratique des biographies historiques n'était pas due à un «goût particulier» de Lucien Febvre, mais à sa théorie de l'histoire proprement dite et à la méthodologie qu'elle implique. Les cuvres de Rabelais et Luther ne sont pas chez Febvre de simples illustrations, mais les véritables lieux ontologiques de manifestation du mental collectif. La continuité établie par Chartier entre les textes théoriques de Febvre et l'anthropologie historique des années soixante est donc problématique - et ce, d'autant plus que les critiques qu'il adresse lui-même à l'histoire quantitative des mentalités pratiquée dans les

33. G. Duby, « Histoire des mentalités », in op. cit., p. 941.

34. R. Chartier, «Histoire intellectuelle et histoire des mentalités. Trajectoires et questions », Revue de synthèse, $\mathrm{n}^{\circ}$ 111-112, juill.-déc. 1983, p. 271-307.

35. Ibid., p. 286.

36. Ibid., p. 294.

37. Ibid., p. 286. 
années 1960-70 s'inscrivent paradoxalement dans la lointaine lignée de Febvre, en ce qu'elles concernent l'impossibilité de résorber l'histoire des idées et l'étude qualitative des œuvres dans la sociologie culturelle.

Alors que Chartier tente d'assimiler l'approche de Febvre à celle de l'anthropologie des années 1960, l'article de Duby s'efforce inversement d'intégrer la perspective anthropologique dans le cadre de la définition febvrienne de l'histoire des mentalités, ce qui provoque tout autant de difficultés. Duby inscrit sa définition de l'histoire des mentalités dans la continuité directe de Febvre. Mais il est difficile de situer «les récents progrès de sciences de l'homme $»^{38}$, et notamment le structuralisme de LéviStrauss $^{39}$ dans le prolongement de la perspective de Febvre, dans la mesure où ils impliquent une représentation de l'homme qui conteste radicalement la pensée humaniste, centrée sur la conscience, de ce dernier. Pour cette raison, le texte de Duby est traversé d'une tension permanente entre la volonté de proposer une perspective qui ne dissolve pas le rôle de l'individu et se maintienne sur le plan de la conscience ${ }^{40}$ et l'effort pour assimiler les développements récents de l'anthropologie, entre une histoire de la diffusion des idées produites par les élites ${ }^{41}$ et l'étude quantitative des schémas conventionnels et des thèmes ou de la structuration sociale des comportements. Cette tension se manifeste notamment par la coexistence d'une définition de l'histoire des mentalités comme histoire du rapport entre individu et groupe, «alternativement, ou mieux simultanément, attentive aux "modèles" culturels et aux réactions personnelles ${ }^{42}$ et la conscience de la «pénurie des documents » ${ }^{43}$ permettant ce type d'études, alors qu'ils deviennent surabondants dès qu'on se situe au niveau des études quantitatives de l'anthropologie culturelle.

Nous avons donc éclairé une des raisons majeures de la confusion propre à la notion d'histoire des mentalités, sans doute responsable de sa disparition. Selon l'angle d'approche adopté, théorique ou pratique, on sera plus enclin à choisir l'une ou l'autre définition de l'histoire des mentalités. La différence de nature entre l'héritage théorique légué par Febvre et la fécondité pratique de l'œuvre de Bloch impliquant leur subsistance sur deux plans distincts, elle a sans doute contribué à rendre invisibles les différences entre ces deux conceptions de l'histoire des mentalités. Or, parce que les

38. G. Duby, « Histoire des mentalités », in op. cit, p. 941.

39. Ibid., p. 962.

40. Ibid., p. 961 : il faut étudier les mythes et croyances «dans le mouvement qui les déplace à travers les différents paliers de la conscience ». Le rituel «fait pénétrer dans les consciences les plus étanches une certaine image de la société ».

41. Ibid., p. 948 : il faut montrer «comment l'ébranlement se propage depuis certains points d'élection ».

42. Ibid., p. 944.

43. Ibid., p. 949. 
défenseurs de ces deux perspectives ont tenté d'imposer une notion générale et exclusive d'histoire des mentalités, ils ont été conduits à occulter les points de divergence, par inclusion forcée de la perspective opposée dans celle qu'ils ont adoptée. Cette volonté de généralisation a donc conduit à transformer l'équivoque de la notion d'histoire des mentalités, oscillant entre deux définitions antagonistes, en une véritable confusion.

\section{2) L'histoire des mentalités, medium d'inscription de l'histoire dans le champ des sciences humaines}

\section{a) L'histoire des mentalités de Febvre et de Bloch : une réponse à la sociologie durkheimienne}

On peut toutefois douter que l'unité du terme « histoire des mentalités » soit exclusivement nominale. Pour que Bloch et Febvre aient utilisé le même terme et que les divergences entre leurs deux perspectives n'aient pas toujours été perçues, ces dernières doivent avoir certains points de convergence, qui doivent pouvoir servir de définition minimale de l'histoire des mentalités. Il s'agit donc de rétablir une notion plus vaste d'histoire des mentalités, englobant deux perspectives distinctes, autrement dit de transformer cette notion équivoque en notion simplement plurivoque.

Quels sont ces éléments communs ? La réponse la plus évidente semble être l'objet étudié : on peut supposer que, à partir de méthodes différentes, les deux auteurs ont entrepris l'étude d'un même domaine encore inexploré de l'histoire humaine. En effet, les thèmes étudiés dans le Livre 2 du tome I de La Société féodale de Bloch, intitulé «Les conditions de vie et l'atmosphère mentale » et les thèmes cités par Febvre dans "Histoire et psychologie ${ }^{44}$ sont globalement les mêmes : l'attachement à la vie, le sens du temps, le sentiment de sécurité, les pratiques alimentaires, le langage, etc. $^{45}$ Mais si on récuse une distinction simpliste entre objet traité et méthodes d'approche - dans la mesure où l'objet étudié est toujours configuré par la pratique discursive qui le vise - cette communauté de domaine doit correspondre à un certain niveau de communauté d'approche.

On peut alors mettre en évidence une convergence négative des deux perspectives. Tout d'abord, l'histoire des mentalités s'oppose à l'anachronisme psychologique, c'est-à-dire à l'interprétation des discours et comportements des hommes du passé d'après les catégories psychologiques du présent. Cette incapacité à se représenter le passé dans sa différence a pour corollaire l'imposition de catégories a priori (Renaissance, Humanisme, Réforme, etc.) sur l'évolution des pensées et des sensibilités

44. In op. cit.

45. Cf. A. Burguières, «La notion de "mentalité" chez Marc Bloch et Lucien Febvre : deux conceptions, deux filiations », in op. cit., p. 342-343. 
humaines. Contre cette perspective, l'histoire des mentalités entend retrouver la spécificité de chaque système de représentations du passé. L'histoire des mentalités s'oppose ensuite à la traditionnelle histoire des idées, qui fait de l'évolution des idées un processus sui generis : les idées y sont produites par d'autres idées, constituant ainsi une trame autonome, qui permet de se dispenser de l'étude de leurs contextes socio-économiques d'apparition. La promotion de l'histoire des mentalités correspond donc à une volonté de retrouver les rapports complexes qui unissent les représentations à leur substrat social, économique et technique.

Mais ces deux oppositions, communes à Febvre et à Bloch, ne tracent finalement que l'abscisse et l'ordonnée de la nouvelle conception de l'histoire promue par les Annales, autrement dit de la définition de l'histoire comme science humaine. Les époques historiques sont présentées comme des unités spécifiques, à l'image des sociétés étudiées par l'ethnologie, et chacune doit être considérée comme un réseau social complexe, dans lequel idées et affects sont étudiés à la mesure de leur poids social. L'attention apportée à de nouveaux domaines de l'étude historique n'est donc finalement que la manifestation secondaire d'une modification structurelle de la science historique. L'invention de l'histoire des mentalités correspond à la volonté de plier l'histoire aux conditions de scientificité définies par Durkheim, qui déniait à l'histoire le statut de science humaine en arguant de son caractère non comparatif ${ }^{46}$. Affirmer que l'histoire est constituée de mentalités strictement distinctes les unes des autres permet ainsi de retrouver la distance de l'ethnologue avec son objet d'étude. Il semble donc que, comme l'affirme André Burguières ${ }^{47}$, la promotion de l'histoire des mentalités ait participé d'une stratégie d'occultation du débat théorique sur la nature de la discipline historique, par l'imposition de fait d'une pratique de l'histoire qui se conforme aux exigences de scientificité produites par la sociologie, en explorant tous les nouveaux domaines de la réalité sociale et proposant des études comparatives. L'indétermination de la notion d'histoire des mentalités, patente dès lors que l'on cherche à spécifier ses méthodes ou son domaine spécifique, se résorbe donc si on comprend qu'elle ne désigne finalement pas autre chose qu'une nouvelle conception de la science historique en général.

\section{b) L'histoire des mentalités des années 1960 : une réponse au développement de l'ethnologie et de l'anthropologie}

Cette première définition de l'histoire des mentalités ne permet toutefois pas de comprendre le succès de cette notion dans les années 1960, alors même que le statut de science humaine de l'histoire était assuré. On peut donner deux raisons à ce renouveau de l'histoire des mentalités.

46. E. Durkheim, Textes 1, Paris, Minuit, 1975, p. 196 sq.

47. A. Burgières, «La notion de "mentalité" chez Marc Bloch et Lucien Febvre : deux conceptions, deux filiations », in op. cit., p. 333. 
Tout d'abord, l'indétermination du contenu de la notion a permis de réadapter sa fonction stratégique d'insertion dans le champ des sciences humaines au nouveau contexte théorique. En effet, les années soixante marquent le renouveau des sciences sociales, l'apparition du structuralisme, un essor de l'anthropologie et de l'ethnologie, qui remet en cause la primauté intellectuelle et institutionnelle de l'histoire. Comme l'a montré Roger Chartier $^{48}$, le développement massif de l'histoire des mentalités à cette période correspond à une stratégie d'annexion des domaines et méthodes des autres sciences humaines. C'est dans ce cadre que l'on doit comprendre la diversité des recherches englobées sous le terme d'histoire des mentalités. De plus, ce contexte permet de rendre raison de la caractérisation de l'histoire des mentalités comme «histoire-carrefour ${ }^{49}$. Elle doit être comprise comme affirmation volontariste de la capacité de l'histoire à produire la synthèse des autres disciplines ${ }^{50}$ et non comme désignation d'une méthode précise de synthèse de ces différents apports.

La seconde raison se situe à la fois en continuité et en contradiction avec la première. Le développement massif de l'histoire des mentalités pendant les années 1960 exprime la volonté des historiens de s'émanciper du carcan de l'histoire économique et sociale, qui avait dominé de façon écrasante les deux décennies précédentes. Les mentalités désignent alors, par opposition, ce qui, dans l'histoire, n'est pas réductible au déterminisme économique et social. La crise du marxisme ainsi que l'effritement de la croyance en l'irréversibilité du progrès économique et technique sont fréquemment évoqués comme justifications de l'essor de l'histoire des mentalités à cette période $^{51}$. Les «mentalités » sont donc investies d'un sens nouveau, propre à cette époque de remise en question de l'hégémonie du déterminisme économique et technique : elles désignent précisément ce qui y résiste. Cette seconde explication peut sembler s'inscrire dans une continuité parfaite avec la première, dans la mesure où ce sont les mêmes raisons qui sont souvent évoquées pour justifier l'apparition du structuralisme et le développement de l'ethnologie à cette période. Que ce soit chez les historiens, les sociologues ou les ethnologues, les intellectuels des années 1960 sont attentifs aux structures de longue durée, à ce qui résiste au changement, aux croyances traditionnelles qui semblent plus profondes que les idéologies. Mais en même temps, cette continuité d'inspiration n'en laisse pas moins subsister une contradiction méthodologique. D'un côté, en effet, l'histoire des

48. R. Chartier, «Histoire intellectuelle et histoire des mentalités. Trajectoires et questions », in op. cit., p. 290.

49. Ibid., p. 106.

50. Cf. J. Le Goff, «Les mentalités. Une histoire ambiguë », in J. Le Goff et P. Nora (dir.), Faire de l'histoire III. Nouveaux objets, Paris, Gallimard, 1974, p. 108-109: «Proche de l'ethnologue, l'historien des mentalités doit se doubler d'un sociologue ». "L'historien des mentalités se rencontre tout particulièrement avec le psychologue social ».

51. J. Revel, «Mentalités », in A. Burguières (dir.), Dictionnaire des sciences historiques, op. cit., p. 454 ; ou P. Ariès, «L'histoire des mentalités », in J. Le Goff (dir.), La Nouvelle histoire, op. cit., ou M. Vovelle, Idéologies et mentalités, Paris, Gallimard, 1992. 
mentalités désigne la capacité de l'histoire à faire la synthèse des développements actuels des sciences humaines, à englober dans une perspective diachronique tous les développements récents de l'étude synchronique des sociétés. Mais de l'autre, elle désigne l'étude de ce qui résiste au changement, des structures immobiles, qui tendent à remettre en question la perspective historiciste sur l'humain. Témoin de cette contradiction, l'article de Jacques Le Goff consacré à la définition de l'histoire des mentalités affirme tout à la fois que l'histoire des mentalités «se situe au point de jonction de l'individuel et du collectif, du temps long et du quotidien, de l'inconscient et de l'intentionnel, du structural et du conjoncturel, du marginal et du général $»^{52}$, et que le terme de mentalité «recouvre un au-delà de l'histoire ${ }^{53}$. L'histoire des mentalités oscille ainsi entre la revendication militante d'un projet d'annexion de l'anthropologie à l'histoire et l'aveu masqué d'une dissolution de l'histoire dans l'anthropologie. L'histoire des mentalités pense pouvoir concilier l'ambition totalisante de Febvre avec la perspective anthropologique de Bloch, tout en oubliant que le principe synthétique de l'histoire des mentalités, pour Febvre, était la conscience individuelle, instance à laquelle le structuralisme dénie toute primauté.

Et de fait, c'est finalement l'ambition de synthèse qui est sacrifiée. L'histoire des mentalités développée pendant les années 1960-70 est très majoritairement une histoire quantitative de la culture matérielle. Les sources traditionnelles de l'histoire sociale (archives notariales, etc.), ainsi que ses méthodes (analyse sérielle, analyse lexicométrique, etc.) sont réutilisées. Cette histoire suppose que les mentalités soient directement appréhendables par l'analyse d'objets (livres ou images), ou de pensées collectives conçues comme des réalités objectives, c'est-à-dire réduites à un ensemble de groupes lexicaux ou d'expressions dont il suffit d'étudier la fréquence. De plus, ces études sérielles des objets culturels suivent les découpages sociologiques déjà produits par l'histoire économique des décennies précédentes, qu'elles permettent donc seulement d'enrichir et non de modifier. Le bilan des travaux réalisés par l'équipe de François Furet sur le rôle du livre dans la France du XVIII ${ }^{\mathrm{e}}$ siècle, publié en $1970^{54}$, est à cet égard éclairant. La première partie se compose d'une séries d'études de « sociologie culturelle », consistant principalement en une analyse statistique des livres achetés ou recensés dans des revues, en fonction de catégories préétablies de livres (ouvrages religieux/scientifiques/littéraires, etc.) et du public supposé de chaque revue (élite parisienne, élite européenne, haute administration française, etc.). La seconde partie, intitulée «sémantique historique », comprend une série d'études quantitatives sur les mots et groupes lexicaux apparaissant fréquemment dans les titres de livres publiés

52. J. Le Goff, « Les mentalités. Une histoire ambiguë », in op. cit., p. 111.

53. Ibid., p. 107.

54. F. Furet (dir.), Livre et société dans la France du XVIII ${ }^{e}$, t. II, Mouton \& Co, Paris-La Haye, 1970. 
pendant la période. Dans les deux cas, la prise en compte de la réalité des livres se réduit à celle de leurs caractéristiques extérieures : titre, genre et auteur. Les statistiques portant sur les «objets culturels » ainsi définis ne sont accompagnées que de considérations a priori sur leur public. Cette histoire plus descriptive qu'explicative a en conséquence beaucoup de mal à rendre raison des changements de mentalités.

À partir de l'unité de la notion d'histoire des mentalités que nous avons établie, celle d'un principe stratégique d'intégration de l'histoire dans le champ évolutif des sciences humaines, les contradictions de la notion apparaissent comme des tensions fécondes, résultant de débats théoriques non résolus. À la tension majeure, issue de l'opposition entre l'approche de Bloch et celle de Febvre, c'est-à-dire entre l'approche psychologique (centrée sur la conscience) et l'approche anthropologique (étudiant une logique sociale qui échappe à la conscience), viennent alors s'ajouter deux autres, plus spécifiques aux années 1960. La première résulte de l'opposition entre la méthode quantitative (étude statistique des objets culturels) qui domine de fait, et la méthode qualitative (étude des œuvres dans leur singularité) qui subsiste au second plan et reste revendiquée par les textes théoriques des historiens des mentalités ${ }^{55}$. La seconde tension s'insinue entre l'ambition synthétique et totalisante de l'histoire des mentalités d'un côté et sa volonté de se constituer comme domaine spécifique, d'isoler son objet et ses méthodes propres de l'autre. Cette reconstruction de la notion d'histoire des mentalités nous permet désormais d'interroger à nouveaux frais la pertinence des critiques qui lui ont été adressées. Nous allons à présent examiner l'ouvrage considéré comme la critique la plus radicale et systématique de l'histoire des mentalités : Pour en finir avec les mentalités de G. E. R. Lloyd ${ }^{56}$.

\section{3) L'histoire des mentalités face à ses critiques}

\section{a) Critique de la fonction explicative de notion de mentalité}

Lloyd concentre son attaque de la notion de mentalité sur son usage dans l'histoire des sciences grecques, qui est son domaine de spécialité. L'ouvrage a toutefois pour ambition de proposer une critique générale de l'usage du concept de mentalité en sciences humaines. S'il ne consacre aucune étude aux travaux des historiens français des mentalités, leurs textes théoriques sont cités dans l'introduction et explicitement présentés comme des exemples typiques de ce qu'il entend critiquer.

55. Cf. par exemple J. Le Goff, «Les mentalités. Une histoire ambiguë », in op. cit., p. 120 : les œuvres littéraires et artistiques sont présentées comme les «sources privilégiées » de l'histoire des mentalités.

56. G. E. R. Lloyd, Pour en finir avec les mentalités, op. cit. 
Lloyd reproche tout d'abord à la notion de mentalité d'être à la fois, et confusément, explicative et descriptive. Ce reproche inclut deux critiques : celle du mode d'explication ethnocentrique proposé par la notion de mentalité, et, plus généralement, de l'ambition explicative d'une notion qui devrait être seulement descriptive.

La critique des implications ethnocentriques de la notion de mentalité proposée par Lloyd consiste simplement à affirmer que celle-ci s'inscrit dans la continuité théorique de la notion lévy-bruhlienne de «mentalité primitive », qui en est l'origine terminologique. Certes, on trouve parfois des traces de cette conception dans l'histoire des mentalités. Ainsi Lucien Febvre présente-t-il, dans son Rabelais, l'outillage mental du XVI ${ }^{\mathrm{e}}$ siècle comme «déficient ${ }^{57}$ par rapport à celui de la rationalité moderne. Chez les historiens des années 1960, dans la mesure où les mentalités sont définies comme ce qui «résiste» aux déterminismes socio-économiques, elles tendent à être pensées comme les traces d'une pensée archaïque dans un monde plus rationnel ${ }^{58}$. Toutefois, l'analyse que nous avons opérée ici du concept de mentalité, sans jamais avoir besoin de faire intervenir celui de «mentalité primitive », révèle que leur recoupement n'est pas systématique, et reste finalement marginal.

Cette assimilation de tout usage de la notion de mentalité à la perspective de Lévy-Bruhl permet à Lloyd d'affirmer que la description de «mentalités» implique nécessairement une explication de l'évolution historique par une évolution de «l'esprit» humain, c'est-à-dire une évolution psychologique voire biologique des structures de la pensée humaine. Formulée de cette façon, il n'est pas besoin de longs discours pour «démystifier» les mentalités, comme le propose le titre original ${ }^{59}$. Elles rendraient raison des phénomènes historiques concrets par une théorie psychologique a priori, fondée sur une représentation ethnocentrique de la rationalité. Elles ne pourraient donc constituer qu'un obstacle à la compréhension effective des phénomènes historiques.

Toutefois, nous avons vu que la cohérence de la notion d'histoire des mentalités ne tenait qu'à sa fonction d'outil d'insertion de l'histoire dans le champ des sciences humaines. Sur cette base commune, les travaux englobés sous le terme d' «histoire des mentalités » ont mobilisé des schémas explicatifs divers. La distinction, présentée plus haut, entre les conceptions psychologiques et sociologiques des mentalités, respectivement rattachées aux filiations de Febvre et de Bloch, permet alors de spécifier le point d'application de la critique de Lloyd : la notion psychologique de mentalité

57. Il s'agit de savoir «quelle efficacité peut avoir la pensée d'hommes qui ne disposent pas des mots philosophiques de base dont l'absence implique vraiment déficience ou lacune de la pensée », L. Febvre, Rabelais ou le problème de l'incroyance au XVI siècle, op. cit., p. 328.

58. Cf. par exemple J. Le Goff, «Les mentalités. Une histoire ambiguë », in op. cit., p. 113 : Lorsque, dans le comportement du Pape Grégoire le Grand, «l'habitude barbare l'emporte sur la règle » monastique, on doit en conclure que « la mentalité a vaincu la doctrine ».

59. G. E. R. Lloyd, Demystifying Mentalities, op. cit. 
est la seule à pouvoir être concernée. La tendance à faire valoir la description des mentalités comme principe d'explication ultime est particulièrement nette dans l'œuvre de Philippe Ariès, qui a notamment eu pour objectif de donner « une interprétation psychologique de la révolution démographique ${ }^{60}$. La forte baisse des taux de natalité et de mortalité au début du XVIII siècle ne doit pas, selon Ariès, être expliquée par des changements techniques (progrès de la médecine) ou des évolutions sociales (perte d'influence de l'idéologie religieuse). Au contraire, c'est parce qu'il y a eu un changement de mentalité que l'idéologie religieuse a perdu sa force, que les techniques contraceptives ont été utilisées, et même que des découvertes médicales ont été faites. "On peut presque dire que le médecin précède la médecine ${ }^{61}$, autrement dit que le besoin psychologique de guérison a précédé les recherches scientifiques. Dans ce modèle, la mentalité tend à être assimilée à une forme d'inconscient collectif, qui se présente alors comme le principe suprême de l'explication historique. Mais cette position théorique avait déjà été critiquée au sein même de l'histoire des mentalités, par exemple par André Burguières ${ }^{62}$. Lloyd ne modifie donc pas réellement les termes du débat.

L'appel à la mentalité, conçue comme une manifestation spécifique de la psychologie collective, a donc certes parfois servi à couper court à l'explication historique. Nous avons toutefois montré que la notion de mentalité ne s'épuisait pas dans cette notion psychologique. Lorsque les mentalités désignent la dimension sociale des représentations - une réalité à expliquer et non un principe explicatif - elles se dérobent alors à cette critique de Lloyd.

\section{b) Critique de la pertinence descriptive de la notion de mentalité}

Nous avons mis en évidence les limites de la critique de la fonction explicative de la notion de mentalité. Mais la «démystification» de Lloyd vise aussi, plus généralement, la pertinence descriptive de la notion de mentalité, autrement dit, selon sa propre définition, l'existence de caractéristiques structurelles, collectives et non conscientes, de la pensée humaine dans une époque et une société (ou même un groupe social) déterminé ${ }^{63}$. Lloyd propose de substituer à l'histoire des mentalités une analyse des «styles d'enquête ${ }^{64}$, c'est-à-dire des modes d'expressions et de conceptualisations utilisés dans un certain contexte de communication,

60. P. Ariès, «Attitudes devant la vie et devant la mort du $\mathrm{XVII}^{\mathrm{e}}$ au $\mathrm{XIX}^{\mathrm{e}}$ siècle », in Population, Revue de l'Institut national d'Études Démographiques, n 3, juil.-sept. 1949, p. 464.

61. P. Ariès, «Attitudes devant la vie et devant la mort du XVII ${ }^{\mathrm{e}}$ au XIX ${ }^{\mathrm{e}}$ siècle », in op. cit., p. 467.

62. Par exemple, A. Burguières, «L'anthropologie historique », in op. cit., p. 156.

63. Ibid., p. 15.

64. G. E. R. Lloyd, op. cit., p. 22. 
lui-même inséré dans un certain contexte social et politique. Il montre ainsi que l'apparition d'un nouveau type de rationalité dans les sciences et la philosophie de l'Athènes du $\mathrm{V}^{\mathrm{e}}$ siècle, caractérisé par une forme argumentative et polémique, ne devrait pas être conçu comme l'expression d'une nouvelle mentalité, mais comme «l'autodéfinition d'un style d'enquête ${ }^{65}$. Les Grecs auraient moins inventé un nouveau mode de connaissance rationnelle que de nouvelles catégories polémiques, dont un des éléments majeurs serait justement l'opposition entre science et mythe. Il s'agirait donc moins d'un renouvellement complet des méthodes et contenus de la connaissance que d'une nouvelle présentation rhétorique de la connaissance comme scientifique et donc radicalement différente de ce qui la précède. Ce choix du concept de "style d'enquête » manifeste déjà un décalage avec l'histoire des mentalités des Annales, dans la mesure où il ne peut être utilisé que pour les discours à prétention explicative et non pour la littérature par exemple. Reste à savoir si, dans ce domaine restreint, la notion permet de résoudre les difficultés posées par celle de mentalité. On comprend la fécondité de cette démarche en ce qui concerne la critique de la notion de mentalité primitive : lorsqu'on oppose radicalement la période archaïque et la période scientifique, on reste prisonnier de la rhétorique de la rationalité utilisée par cette seconde période, alors qu'elle est en réalité sa principale «création».

Mais si, au lieu de comparer la thèse de l'apparition spontanée d'une mentalité «scientifique » avec la thèse de l'autodéfinition d'un nouveau style d'enquête, on se contente de comparer l'idée de mentalité en général avec celle de "style d'enquête », quelle différence subsiste-t-il entre les deux notions ? Le concept de «style d'enquête » présente trois avantages. Tout d'abord, la mise en évidence du caractère rhétorique de l'opposition entre période mythique et période rationnelle a pour effet de minorer leur différence effective : l'écart dont on doit rendre raison est plus faible que les textes de la seconde période ne semblaient l'indiquer. Il y a donc une réduction de l'objet à expliquer. Ensuite le style d'enquête étant susceptible d'une auto-définition par ses utilisateurs, on n'a pas besoin de postuler l'existence de croyances ou de modes de pensée non conscients. Enfin, dans la mesure où le «style d'enquête» est spécifique à certains contextes d'utilisation, il ne désigne pas une caractéristique structurelle de la pensée des individus, mais un simple mode de discours parmi d'autres. Toutefois, la notion de «style d'enquête » n'évacue pas la dimension de cohérence impliquée dans la notion de mentalité, puisqu'elle postule l'invention de nouvelles formes d'expression, valables pour tout individu désirant utiliser ce mode de discours. Par exemple, pour pouvoir utiliser le mode «philosophique» du discours, il faudra utiliser une forme argumentative qui n'existait pas auparavant. Même si la coupure entre les deux périodes («archaïque » et «scientifique ») n'est pas aussi radicale qu'on ne l'a cru, 
elle est donc bien réelle, puisque cette illusion est le produit d'une stratégie spécifique des membres de la seconde période, ou du second groupe.

Comment peut-on alors expliquer l'apparition d'un nouveau style d'enquête ? Afin d'éviter de reproduire la confusion qu'il dénonçait, dans la notion de mentalité, entre description et explication, Lloyd propose de n'utiliser la notion de «style d'enquête » qu'à des fins descriptives. Quant à l'explication de cette mutation, elle devra être trouvée dans son lien avec les transformations socio-politiques de l'époque. Lloyd reprend alors à son compte la thèse de Jean-Pierre Vernant selon laquelle la philosophie grecque serait la «fille de la Cité », c'est-à-dire issue du contexte politique de la démocratie athénienne. Il faudrait donc remplacer l'hypothèse d'une succession de mentalités autonomes par l'analyse d'entités linguistiques appelés «styles d'enquête », dont les transformations seraient dérivables de mutations dans le contexte socio-politique. Ainsi, à Athènes, l'existence d'un débat politique ouvert à tous les citoyens, tout comme la possibilité corrélative de refuser les arguments d'autorité, auraient rendu envisageable la remise en question des connaissances traditionnelles sur la nature, tout comme l'invention de la philosophie en tant que forme de pensée basée sur l'argumentation et la présence de la raison en tout homme. La thèse de Lloyd soulève toutefois deux difficultés.

Premièrement, rendre raison de la rationalité de la science et de la philosophie grecque par l'existence d'un contexte démocratique - et de la possibilité de remise en question de toutes les opinions qu'il suppose - ne revient-il pas idéaliser le fonctionnement de démocratie grecque, bien loin d'être dans les faits pur de toute lutte de pouvoir, d'influences et d'autorité ? Pour résoudre ce problème, Lloyd souligne que, quand bien même la pratique politique grecque s'éloignait de son idéal, «cela ne diminuait en rien l'importance de cette image en tant qu'indice de ce que l'on croyait, ou du moins que l'on concevait comme idéal $»^{66}$. Mais alors, il ne s'agit plus d'une détermination des modes de pensée (philosophiques et scientifiques) par la pratique (politique), mais par un idéal (politique), c'est-à-dire une représentation collective. Au lieu de dériver les modes de pensée d'une réalité plus concrète, une pratique politique incarnée dans des institutions, on les dérive d'une réalité encore plus abstraite qu'eux : un idéal. La pratique théorique des philosophes et scientifiques est donc une réalisation, une incarnation de l'idéal démocratique dans une pratique (théorique) effective : ils transforment l'idéal d'argumentation en pratique de l'argumentation, l'idéal de remise en question des opinions en remise en question effective des savoirs traditionnels. Dès lors, le problème de l'origine des représentations, et plus précisément de l'idéal démocratique, redevient central.

Deuxièmement, Lloyd se refuse à faire de l'explication socio-politique un déterminisme strict. Il n'a de cesse d'affirmer que cette causalité

66. G. E. R. Lloyd, Pour en finir avec les mentalités, op. cit., p. 22. 
reste partielle. En plus des rapports d'influence, on peut «indiquer des parallélismes entre certains éléments de l'enquête intellectuelle et certains aspects de la vie politique ${ }^{67}$, (notamment des similitudes de formes). Mais la présence de «parallélismes » entre l'activité démocratique et la pensée philosophique et scientifique en Grèce ancienne implique que l'on puisse abstraire de ces pratiques différentes des éléments communs, en particuliers formels. Quel sera alors le statut théorique de ces formes communes, dont l'existence ne peut être justifiée par une relation de causalité ou d'influence? Ne doit-on pas en déduire l'existence d'une forme, au moins minimale, d'invariants structurels, propre à la pensée ou aux discours de l'époque?

La notion de style d'enquête laisse ainsi deux problèmes en chantier : l'existence de traits parallèles entre des pratiques ou des «modes de pensée » distincts, ainsi que l'origine des idéologies. Or, ce sont ces difficultés que tente précisément de traiter la notion de mentalité. En effet, cette dernière se caractérise notamment par la «focalisation sur la structure des croyances et leurs rapports mutuels ${ }^{68}$, alors que la notion de style d'enquête met l'accent sur l'hétérogénéité des modes de discours, rapportée à la diversité des contextes de communication. Mais la découverte de «parallélismes» entre les activités démocratiques, philosophiques et scientifiques ne nous conduit-elle pas à reconnaître l'existence de modes de pensée ou de discours invariants, permettant ainsi d'accorder à la description de traits structuraux, opérée par la notion de "mentalité », une certaine pertinence? De plus, ces «parallélismes » sont observables au seul niveau abstrait de la comparaison entre les différents discours et pratiques et non à celui de la conscience des acteurs. Ces «parallélismes » correspondent donc précisément à ce que les historiens ont désigné par le terme de mentalités : les traits communs aux pensées, croyances, affects des hommes d'une époque ou d'une société, qui résistent à l'explication par une causalité socio-politique. En effet, les historiens des Annales n'ont jamais eu pour ambition de substituer aux explications sociologiques une explication par les mentalités : même dans sa version psychologique la plus caricaturale, le terme désigne toujours ce qui outrepasse le déterminisme sociologique, jamais ce qui le remplace entièrement. Dans l'exemple de Jacques Le Goff précédemment cité, la «mentalité religieuse » n'est évoquée que dans la mesure où les causes socio-économiques de la croisade «n'expliquent pas tout ${ }^{69}$.

Lloyd contourne ces difficultés en ne traitant que de l'interprétation d'énoncés isolés. À la fin de l'ouvrage, Lloyd reconnaît alors avoir laissé pour compte la question synchronique (des «différences et corrélations des modes de raisonnements dans les différents domaines du discours $\gg^{70}$ ) ainsi

67. Ibid., p. 96.

68. Ibid., p. 5.

69. J. Le Goff, « Les mentalités. Une histoire ambiguë », in op. cit., p. 107.

70. G. E. R. Lloyd, Pour en finir avec les mentalités, op. cit., p. 219. 
que la question diachronique («la manière dont ils sont susceptibles de se modifier $»^{71}$ ). Il justifie cette méthode en affirmant que, une fois l'argument exposé au niveau minimal de l'énoncé, il ne reste qu'à l'étendre au niveau global. De même qu'on ne peut rendre raison des énoncés sans rendre raison de leur contexte de communication, «de même on peut dire plus généralement qu'on ne peut répondre aux questions relatives aux systèmes de croyances ou aux modes de raisonnement dans leur ensemble que si l'on s'attache aux types d'interactions sociales et aux attentes que les participants peuvent entretenir sur la nature et la manière de les conduire $»^{72}$. Mais il ne viendrait à l'idée d'aucun historien de remettre en cause la nécessité de rattacher les pensées et croyances au contexte social dans lequel elles s'insèrent. Ces notions de «systèmes de croyances» et de «modes de raisonnement dans leur ensemble », qui seules seraient en mesure d'être comparées avec la notion de mentalité, ne sont ni développées ni même explicitées. De plus, Lloyd prétend pouvoir ramener tous les phénomènes étudiés par l'histoire des mentalités sur le seul plan du discours. Or, l'histoire des mentalités a étudié des phénomènes qui n'ont tout simplement pas d'existence sur ce plan. On peut reprendre l'exemple de la découverte de l'emploi massif et commun à toutes les classes sociales des techniques contraceptives à partir du début du XVIII ${ }^{\mathrm{e}}$ siècle, alors que ces dernières étaient connues depuis longtemps sans être utilisées ${ }^{73}$ : ce phénomène global, qui a été mis au jour par des statistiques des naissances, n'était pas perçu par les contemporains. Il ne peut donc tout simplement pas être ramené au plan du discours et des stratégies conscientes.

Les arguments de Lloyd ne permettent donc pas réellement de remettre en cause la démarche propre à l'histoire des mentalités. La critique de la confusion entre explication et description ne l'atteint que très marginalement, et la notion descriptive qu'il propose de substituer à celle de mentalité n'est tout simplement pas du même ordre, puisqu'elle ne prend pas en compte les phénomènes d'ensemble, diachroniques ou synchroniques, ainsi que les phénomènes non linguistiques. Cette «démystification » des mentalités ne produit finalement pas autre chose que la substitution, à la notion ethnocentrique de mentalité primitive, d'une notion indéterminée de mentalité, en cela très semblable à celle employée par les Annales.

Plutôt qu'un nouveau domaine de recherche, l'histoire des mentalités a donc apporté à l'histoire une nouvelle problématique, celle de son mode d'intégration au champ des sciences humaines, ainsi que deux modalités de réponses : une approche d'ascendance psychologique, issue de l'œuvre de Febvre et une approche d'ascendance sociologique, issue de celle de Bloch.

\section{Ibid.}

72. Ibid.

73. Cf. P. Ariès, L'histoire des populations françaises et leur attitude devant la vie, Paris, Le Seuil, 1948. 
La pluralité des notions qui tentent encore de nos jours de se substituer à celle d'histoire des mentalités (anthropologie historique, histoire culturelle, histoire de l'imaginaire ou des représentations, etc.), sans parvenir à s'imposer durablement, révèle que la problématique que cette dernière a mise en place est toujours pertinente et que le débat entre ses deux options n'a pas été tranché. L'histoire des mentalités a posé le cadre d'une réflexion qui est toujours d'actualité. 